\title{
Robustness of Neural Coding in Drosophila Photoreceptors in the Absence of Slow Delayed Rectifier $\mathrm{K}^{+}$Channels
}

\author{
Mikko Vähäsöyrinki, ${ }^{1}$ Jeremy E. Niven, ${ }^{2}$ Roger C. Hardie, ${ }^{3}$ Matti Weckström, ${ }^{1}$ and Mikko Juusola ${ }^{2}$ \\ ${ }^{1}$ Department of Physical Sciences, Division of Biophysics and Biocenter Oulu, University of Oulu, 90014 Oulun Yliopisto, Oulu, Finland, ${ }^{2} \mathrm{Physiological}$ \\ Laboratory, University of Cambridge, Cambridge CB2 3EG, United Kingdom, and ${ }^{3}$ Department of Anatomy, University of Cambridge, Cambridge CB2 3DY, \\ United Kingdom
}

Determining the contribution of a single type of ion channel to information processing within a neuron requires not only knowledge of the properties of the channel but also understanding of its function within a complex system. We studied the contribution of slow delayed rectifier $\mathrm{K}^{+}$channels to neural coding in Drosophila photoreceptors by combining genetic and electrophysiological approaches with biophysical modeling. We show that the Shab gene encodes the slow delayed rectifier $\mathrm{K}^{+}$channel and identify a novel voltage-gated $\mathrm{K}^{+}$ conductance. Analysis of the in vivo recorded voltage responses together with their computer-simulated counterparts demonstrates that Shab channels in Drosophila photoreceptors attenuate the light-induced depolarization and prevent response saturation in bright light. We also show that reduction of the Shab conductance in mutant photoreceptors is accompanied by a proportional drop in their input resistance. This reduction in input resistance partially restores the signaling range, sensitivity, and dynamic coding of light intensities of $S h a b$ photoreceptors to those of the wild-type counterparts. However, loss of the Shab channels may affect both the energy efficiency of coding and the processing of natural stimuli. Our results highlight the role of different types of voltage-gated $\mathrm{K}^{+}$channels in the performance of the photoreceptors and provide insight into functional robustness against the perturbation of specific ion channel composition.

Key words: vision; nonspiking; information; intracellular; patch clamp; modeling

\section{Introduction}

The activity of a neuron is critically dependent on the suite of voltage-gated ion channels present in its membrane (Hille, 2001). Neurons possess an array of these channels, providing precise control over their electrical excitability and enabling them to process biologically relevant information accurately. Voltagegated $\mathrm{K}^{+}$channels, in particular, are extremely diverse in function (Rudy, 1988; Coetzee et al., 1999), contributing to regulation of distinct aspects of neuronal activity by shaping the voltage responses (Connor and Stevens, 1971; Laurent, 1990; Weckström et al., 1991; Debanne et al., 1997; Hoffman et al., 1997; Niven et al., 2003a; Bernard et al., 2004). Despite their importance in regulating neural activity, genetic deletions of specific ion channels may produce relatively little change in performance, even when

Received Aug. 8, 2005; revised Jan. 16, 2006; accepted Jan. 16, 2006.

This work was supported by the Finnish Academy of Science and Letters (M.V.), the Finnish Academy (M.W. M.V.), the Wellcome Trust (M.J.), The Gatsby Charitable Foundation (M.J.), The Royal Society (M.J.), the Biotechnology and Biological Sciences Research Council (M.J., R.C.H.), and the Medical Research Council (R.C.H.). We thank Simon Laughlin and Doekele Stavenga for comments and suggestions.

${ }^{*} M$.V. and J.E.N. contributed equally to this work.

Correspondence should be addressed to Dr. Matti Weckström, Department of Physical Sciences, Division of Biophysics, and Biocenter Oulu, University of Oulu, P.O. Box 3000, 90014 Oulun Yliopisto, Oulu, Finland. E-mail: matti.weckstrom@oulu.fi.

M. Vähäsöyrinki's present address: Computation and Neural Systems, Division of Biology, California Institute of Technology, Pasadena, CA 91125

J. E. Niven's present address: Department of Zoology, University of Cambridge, Cambridge CB2 3EJ, UK.

M. Juusola's present address: Department of Biomedical Science, University of Sheffield, Sheffield S10 2TN, UK. D0I:10.1523/JNEUROSCI.3316-05.2006

Copyright $\odot 2006$ Society for Neuroscience $\quad$ 0270-6474/06/262652-09\$15.00/0 pharmacological or modeling studies suggest that the phenotypes should be severe (Liu et al., 1998; Namkung et al., 1998; Wickman et al., 1998; Akopian et al., 1999; Brickley et al., 2001; MacLean et al., 2003; Niven et al., 2003a). This robustness may be required to ensure that neurons maintain their response characteristics despite variable levels of channel expression (Goldman et al., 2001; Marder and Prinz, 2002; Niven, 2004). Indeed, recent work has shown that identified neurons in the crab stomatogastric ganglion generate similar outputs despite large fluctuations in $\mathrm{K}^{+}$ conductance densities between individuals (Liu et al., 1998; Golowasch et al., 1999; Prinz et al., 2003).

Because of their different biophysical properties, deletion of specific types of $\mathrm{K}^{+}$channels should produce different functional consequences depending on the properties of both the channel and the neuron. Isolating the contribution of one specific ion channel type to the performance of the neurons and quantifying robustness against changes in these specific channels has been under intensive study in several systems (Marder and Prinz, 2002). However, the results may be complicated by several uncontrolled factors, such as the contribution of other types of ion channels, nonspecificity of pharmacological methods, or the possible disruption of normal neuronal function in vitro. Additional insight might be gained by using a genetically tractable system with relatively simple ion channel composition in addition to easily controllable inputs and outputs, such as Drosophila photoreceptors.

Drosophila photoreceptors express predominately two types 
of voltage-gated $\mathrm{K}^{+}$channels, Shaker and a slow delayed rectifier (Hardie, 1991a). In this study, we identify the Shab gene as encoding the slow delayed rectifier $\mathrm{K}^{+}$channel. Our results indicate that the role of Shab channels in Drosophila photoreceptors is to dynamically attenuate the light-induced depolarization and prevent response saturation in bright light. Comparison of mutant photoreceptors lacking Shab channels with their wild-type (WT) counterparts and with a biophysical photoreceptor model revealed that compensatory changes had occurred after the removal of the Shab channels. By using two other mutations, which reduce but do not eliminate the Shab conductance, we show that a proportional decrease in the steady-state input resistance accompanies the loss of the Shab conductance. This regulation of the electrical properties may act as a mechanism to maintain photoreceptor function despite natural variation in the voltagegated ion channel composition.

\section{Materials and Methods}

Fly stocks. Flies (Drosophila melanogaster) were raised on standard medium at $19^{\circ} \mathrm{C}$ in darkness. The wild-type strain was red-eyed Oregon Red. Three alleles of the Shab gene $\left(S h a b^{1}\right.$, Shab ${ }^{2}$, and $\left.S h a b^{3}\right)$ were used either in ebony or WT background. Shab ${ }^{1}$ and $S h a b^{2}$ are missense mutations, whereas $S h a b^{3}$ is a null allele (Hegde et al., 1999). The Shaker ${ }^{14}$ null allele (a missense mutation in the core region resulting in nonfunctional Shaker channels) and Shaker ${ }^{14}$;Shab ${ }^{3}$ double mutants were also used in red-eyed flies.

Optical neutralization of the cornea. For observing rhabdomeres in the intact eye, heads were mounted on microscope slides using clear nail varnish and were observed under a $63 \times$ oil-immersion objective using antidromic illumination for photography (Franceschini and Kirschfeld, 1971).

Whole-cell recordings. Dissociated ommatidia were prepared as described previously from recently eclosed adult flies (Hardie, 1991b) and were transferred to the bottom of a recording chamber on an inverted Nikon (Tokyo, Japan) Diaphot microscope. The bath was composed of the following (in $\mathrm{mm}$ ): $120 \mathrm{NaCl}, 5 \mathrm{KCl}, 10 \mathrm{~N}$-tris(hydroxymethyl) methyl-2-aminoethane-sulfonic acid (TES), $4 \mathrm{MgCl}_{2}, 1.5 \mathrm{CaCl}_{2}, 25$ proline, and 5 alanine. The standard intracellular solution contained the following (in $\mathrm{mm}$ ): $140 \mathrm{~K}^{+}$gluconate, 10 TES, $4 \mathrm{MgATP}_{2} \mathrm{MgCl}_{2}, 1$ nicotinamide-adenine dinucleotide, and $0.4 \mathrm{Na}^{+}$-GTP. The $\mathrm{pH}$ of all solutions was 7.15 . Whole-cell voltage-clamp recordings were made using electrodes with a resistance of $\sim 10-15 \mathrm{M} \Omega$. The series resistance values were generally below $25 \mathrm{M} \Omega$ and were routinely compensated to $>80 \%$. Data were collected and analyzed using an Axopatch 1-D amplifier and pClamp 8 or 9 software (Molecular Devices, Palo Alto, CA). Cells were stimulated via a green light-emitting diode (LED), with maximum effective intensity of $\sim 2 \times 10^{5}$ photons per second per photoreceptor. Relative intensities were calibrated using a linear photodiode and converted to absolute intensities in terms of effectively absorbed photons by counting quantum bumps at low intensities (Henderson et al., 2000).

In vivo intracellular recordings. In vivo intracellular recordings were performed by placing a quartz glass-capillary electrode into the retina through a small hole cut in the surface of the eye (Juusola and Hardie, 2001). Photoreceptors were stimulated with a high-intensity green LED (Marl Optosource, Ulverston, UK) driven by a custom-built LED driver and monitored with a pin diode circuit. The LED light output was attenuated using neutral density filters (Kodak Wratten; Eastman Kodak, Rochester, NY) to provide 10 adapting light backgrounds in $\sim 0.5 \mathrm{log}$ unit steps. Photoreceptor voltage responses were recorded with a switchclamp amplifier (SEC 10L; NPI Electronics, Tamm, Germany). Photoreceptors were considered for analysis only if their membrane potential was less than $-55 \mathrm{mV}$ and they had at least a $45 \mathrm{mV}$ saturating impulse response in dark-adapted conditions. Data acquisition, stimulus generation, and signal analysis were performed by a purpose built Matlab (MathWorks, Natick, MA) interface (Juusola and Hardie, 2001). Statistical tests were done with the Origin 7.0 software (Microcal Software, Northampton, MA).
Single impaled photoreceptors were stimulated with 10 repetitions of identical pseudorandom white-noise-modulated light contrast (light intensity was modulated around a certain background). Averaging the responses gave an estimate of the noise-free voltage signal, and subtraction of this signal from each individual trace gave an estimate of the noise. Signal-to-noise ratio $[\mathrm{SNR}(f)]$, was calculated by dividing the autospectra of the signal by that of the noise. From the $\operatorname{SNR}(f)$, the information capacity (in bits per second), $C$, was obtained by Shannon's formula:

$$
C=\int_{0}^{\infty} \log _{2}(\operatorname{SNR}(f)+1) d f
$$

where integration was limited to $150 \mathrm{~Hz}$. This method is likely to produce an overestimate of the information capacity (Juusola and de Polavieja, 2003), but resulting systematic error will not affect the comparisons made between the WT and mutant flies.

The photoreceptor frequency response, $G(f)$, was calculated by dividing the cross-spectrum of the input (contrast) and output (averaged photoreceptor voltage signal) with the autospectrum of the input. The frequency response, $G(f)$, was expressed in terms of its gain, the ratio of the photoreceptor response amplitude to the stimulus amplitude.

Modeling. A Hodgkin-Huxley-type model of the photo-insensitive membrane of Drosophila photoreceptors was developed using Matlab software (MathWorks). Derivation and validation of this modeling scheme has been described previously (Niven et al., 2003a). Briefly, the model included Shaker and slow delayed rectifier voltage-gated potassium conductances, in addition to potassium and chloride leak conductances (the fast delayed rectifier conductance was omitted because it is present only in some photoreceptors). The voltage-dependent properties (including time constants and steady-state activation and inactivation functions) for the Shaker and slow delayed rectifier conductances were obtained from published data (Hardie, 1991a; Hevers and Hardie, 1995; Niven et al., 2003a). In addition, a novel voltage-gated potassium conductance was included using the voltage-dependent properties characterized in whole-cell recordings of Shaker ${ }^{14}$;Shab ${ }^{3}$ photoreceptors (see Fig. $3 c, e)$ and following similar procedures as for the Shaker and slow delayed rectifier channels (Niven et al., 2003a). Additionally, the partial failure of Shaker channel inactivation visible in the whole-cell currents of $S_{\mathrm{Sab}}{ }^{3}$ photoreceptors was incorporated.

The voltage-dependent properties of the ion channels, the reversal potentials for each ion, and the membrane area were fixed parameters within the model. Other photoreceptor membrane properties (the maximum values of the voltage-gated conductances, resting potential, leak conductances, and membrane capacitance) were estimated from in vivo current injection experiments by fitting the simulated voltage responses to the experimental ones (see Fig. 2). Maximum conductance of the slow delayed rectifier was set to zero for the $S h a b^{3}$ photoreceptor model (iterated to be $\sim 30 \%$ of that of the WT for the Shab ${ }^{1}$ photoreceptor model). The same maximum conductance values of the Shaker and novel potassium conductances produced good fits with experimental data for the WT, $S h a b^{1}$, or $S h a b^{3}$ models and were used throughout the simulations. This allowed us to exclude the possibility that differences in the properties of each model would be attributable to changes in the Shaker conductance or the novel potassium conductance. In addition to experimentally fitted WT, Shab ${ }^{1}$, or $S h a b^{3}$ photoreceptor models, two additional hypothetical models were used: "WT with $30 \%$ Shab" and "WT without Shab." These were generated using the membrane properties derived from experimental WT cells but setting the maximum conductance of the slow delayed rectifier to $30 \%$ of that of the WT or to zero, respectively. These hypothetical cases simulated the Shab ${ }^{1}$ and Shab $b^{3}$ mutant photoreceptors without accompanying change in the input resistance.

The conductance of the light-dependent channels [light-induced conductance (LIC)] while exposing photoreceptors to the white-noisemodulated light contrast stimulus was reconstructed from the experimental WT voltage response using the photoreceptor model (see Fig. 5b). During light stimulation. the photon flux activates numerous microvilli in the photosensitive membrane, whose activity was modeled as a single average LIC input to the photo-insensitive membrane, with a reversal 
potential of $+10 \mathrm{mV}$ (Reuss et al., 1997; Niven et al., 2004). This LIC was used to drive the model, and its values were iterated at each sample point until the experimental voltage response was reproduced. The resulting LIC was subsequently low-pass filtered (fourth-order Butterworth filter with $500 \mathrm{~Hz}$ corner frequency) to remove the artifactual highfrequency content attributable to fitting noise in the experimental voltage response and random fluctuations below the iteration error bound. Although the predicted LIC will contain residual errors from imperfect modeling of the photo-insensitive membrane, it enabled realistic simulations with experimentally derived input dynamics. The predicted LIC was used in simulations for the experimental (WT, Shab ${ }^{1}$, or $S h a b^{3}$ ) and hypothetical (WT with 30\% Shab and WT without Shab) models.

The current-voltage $(I-V)$ relationships for the models were determined by using 70 evenly spaced, $100 \mathrm{~ms}$ current pulses, ranging from -0.15 to $0.4 \mathrm{nA}$ as an input and sampling the simulated membrane voltage at the end of each pulse.

\section{Results}

Shab encodes the slow delayed rectifier $\mathrm{K}^{+}$channel in Drosophila photoreceptors

Drosophila photoreceptors can be thought to be divided into two interrelated functional compartments: photosensitive and photo-insensitive membrane. Throughout this study, we define the photosensitive membrane as consisting of the convoluted microvillar part of the photoreceptors, which includes the molecular machinery of phototransduction. The photo-insensitive membrane then means the rest of cell body. The photoinsensitive membrane has been shown to be voltage dependent, containing three voltage-gated $\mathrm{K}^{+}$currents: an A-type (Shaker), a slow delayed rectifier, and, in some cells, a fast delayed rectifier (Hardie, 1991a). The A-type and slow delayed rectifier currents are clearly visible in in vitro voltage-clamp recordings of isolated WT photoreceptors as a transient current and a slowly inactivating current, respectively (Fig. 1a). Null mutations of the Shaker gene eliminate the transient $\mathrm{K}^{+}$current but leave the slowly inactivating current intact (Fig. $1 b$ ), establishing that the Shaker gene encodes the A-type $\mathrm{K}^{+}$channels (Hardie et al., 1991; Niven et al., 2003a). The gene encoding the slow delayed rectifier in Drosophila photoreceptors has so far not been identified. Because mutations at the Shab locus are known to affect sustained $\mathrm{K}^{+}$currents in Drosophila muscles (Hegde et al., 1999), we performed voltage-clamp recordings of isolated photoreceptors from $S h a b^{3}$ flies. The results show that the Shaker current was unaffected but the slowly inactivating current was eliminated (Fig. 1c), indicating that the Shab gene encodes the slow delayed rectifier.

To ensure that mutation of the Shab gene did not affect photoreceptor morphology, we illuminated the photoreceptors antidromically to determine their rhabdomere structure and spacing (Fig. 1d). Each $\mathrm{Shab}^{3}$ ommatidium contained a standard config-

$40 \mathrm{mV}$ b

C

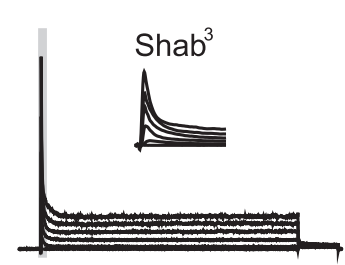

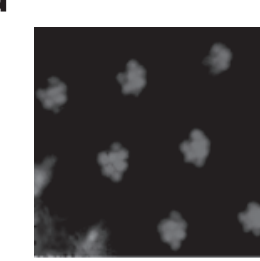

g

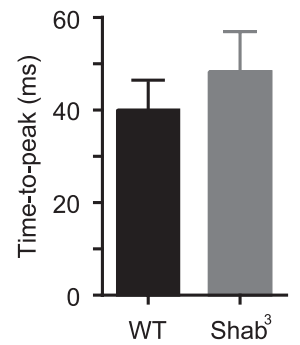

e

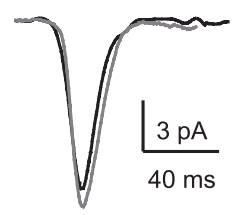

h

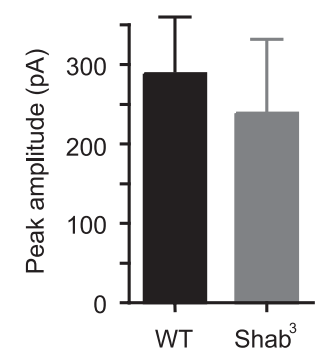

f

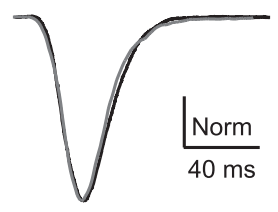

i

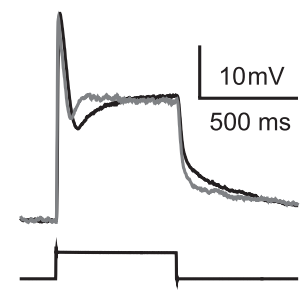

Figure 1. Shab encodes the slow delayed rectifier voltage-gated $\mathrm{K}^{+}$channel in Drosophila photoreceptors. Currents recorded from isolated photoreceptors in vitro using whole-cell patch-clamp: $\boldsymbol{a}, \mathrm{WT} ; \boldsymbol{b}$, Shaker ${ }^{14} ; \boldsymbol{c}$, Shab ${ }^{3}$. Insets, Enlargement of gray region in $\boldsymbol{a}$ and $\mathbf{c}$ showing the rapidly inactivating $\mathrm{K}^{+}$current encoded by the Shaker gene. $\boldsymbol{d}$, Antidromic illumination of Shab brief $\left(1 \mathrm{~ms}\right.$ ) flashes containing $\sim 70$ effective photons delivered at time 0 in WT (black) and Shab ${ }^{3}$ (gray) photoreceptors voltage photons) in WT (black) and Shab ${ }^{3}$ (gray) photoreceptors. Mean \pm SEM; $n=6$ (WT) and 7 (Shab). $i$, Mean in vivo responses to $1 \mathrm{~s}$ light pulse for the WT (black) and Shab ${ }^{3}$ (gray) photoreceptors $(n=6)$.

uration of photoreceptors with normal rhabdoms. Furthermore, in whole-cell recordings, the cell capacitances, which are dominated by the contribution of the microvillar membrane, were indistinguishable from WT $(\sim 65 \mathrm{pF})$, as was the appearance of dissociated ommatidia. Shab mutations could also potentially affect the properties of the phototransduction machinery, leading to altered light-induced currents in the mutant photoreceptors. To exclude this possibility, we compared bumps (elementary responses of the phototransduction machinery corresponding to single-photon absorptions) and impulse responses to light flashes ( $\sim 70$ photons/s) of Shab $b^{3}$ photoreceptors with those of WT photoreceptors under voltage clamp in the whole-cell configuration (Fig. 1e,f). Bump waveforms were similar in both WT and $S h a b^{3}$ photoreceptors (Fig. 1e). The macroscopic kinetics, assessed by time-to-peak and amplitude of the impulse response, of $S h a b^{3}$ photoreceptors were also not significantly different from those of WT photoreceptors (Fig. 1g,h).

Insect photoreceptors operate over a vast range of light intensities up to $\sim 10^{7}$ photons/s (Howard et al., 1987). However, photoreceptors in vitro do not survive prolonged exposure to high light intensities. Therefore, we performed in vivo intracellular experiments enabling us to record photoreceptor light re- 
sponses over the whole dynamic range (Juusola and Hardie, 2001; Niven et al., 2003a). To get a qualitative idea of the effect of deleting Shab channels on the intact photoreceptors, we recorded voltage responses to saturating light pulses in Shab $b^{3}$ mutants. The responses showed surprising robustness when compared with those of WT photoreceptors, even after the initial transient when the activation of Shab channels would normally contribute to membrane repolarization (Fig. 1i).

\section{Photo-insensitive membrane properties of WT and Shab mutant photoreceptors}

Robustness of the in vivo light response in $\mathrm{Shab}^{3}$ (Fig. 1i) suggests that eliminating the Shab conductance does not compromise photoreceptor function. Loss of the Shab conductance should, however, affect the electrical properties of Drosophila photoreceptors. To study this, we developed a mathematical model of the photo-insensitive membrane based on Hodgkin-Huxley-type equations that included both the Shaker and Shab conductances (see Materials and Methods). Simulated voltage responses of WT photoreceptors to positive and negative current steps matched closely those of Drosophila photoreceptors recorded in vivo (Fig. $2 a, b)$. Both the in vivo recordings and the model simulations showed characteristic voltage-dependent properties (Fig. 2a,b), including a gradual amplification generated by Shaker channel inactivation (Niven et al., 2003a).

What effect does the deletion of the Shab channels, as occurs in $S h a b^{3}$ mutant photoreceptors, have on the simulated voltage responses from the model? To assess the effect, we removed the Shab conductance from the WT model and resimulated the current injection experiments (Fig. 2c). The resulting responses were clearly different from those of either the WT model responses or those recorded in vivo from WT photoreceptors (Fig. $2 a-c$ ). Surprisingly, the predicted voltage responses of the model without the Shab conductance were also different from the voltage responses recorded in vivo from Shab ${ }^{3}$ photoreceptors (Fig. $2 c, d$ ): positive current steps evoked much larger depolarizing responses in the model prediction than in the in vivo from Shab $b^{3}$ photoreceptors. Moreover, voltage responses evoked by negative current steps indicated that input resistance of the $S h a b^{3}$ photoreceptors was significantly decreased relative to the WT photoreceptors (Fig. 2a,d). Can this difference in passive membrane properties account for the failure of the model to simulate experimental $S h a b^{3}$ photoreceptor responses? Although fitting the passive membrane properties of the model reproduced the Sha $b^{3}$ hyperpolarizing voltage responses (reduced input resistance was simulated by increasing the leak conductances in the model), there was still a considerable discrepancy between the depolarizing ones (Fig. $2 d, e$ ). This discrepancy suggested that an additional voltage-gated conductance might be present in the photoinsensitive membrane of Drosophila photoreceptors.

To detect an additional voltage-gated conductance in the photo-insensitive membrane of Drosophila photoreceptors, we generated a Shaker ${ }^{14}$;Shab ${ }^{3}$ double mutant that eliminates the two most prominent voltage-gated conductances. These flies were poorly viable and infertile; however, a small percentage of adults emerged from a balanced heterozygote stock, allowing whole-cell patch-clamp recordings to be made of photoreceptors lacking both Shaker and Shab $\mathrm{K}^{+}$channels. In some Shaker ${ }^{14}$; $S h a b^{3}$ double-mutant photoreceptors, the fast delayed rectifier voltage-gated $\mathrm{K}^{+}$current was present, showing that it is not encoded by the Shab gene (Fig. $3 a$ ). However, an additional novel slowly activating, non-inactivating potassium current was also present in all Shaker ${ }^{14}$;Shab ${ }^{3}$ photoreceptors (Fig. 3a) and, in a

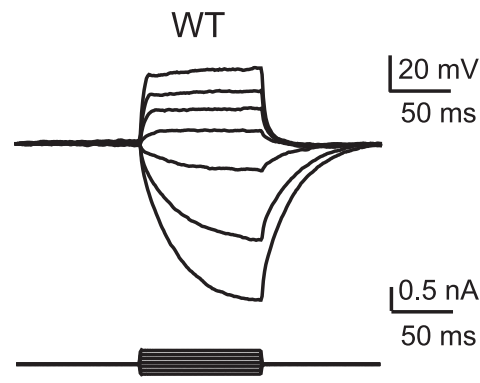

b

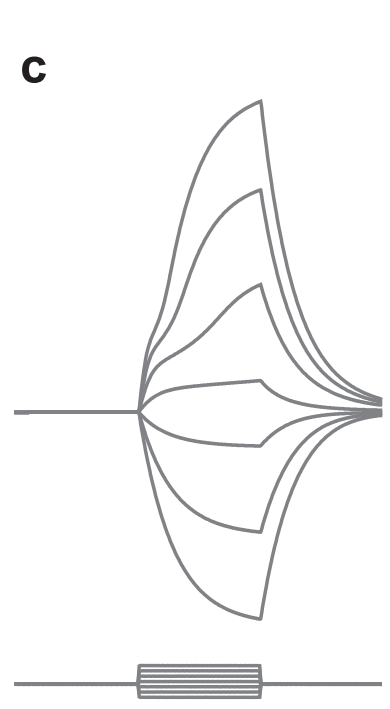

d

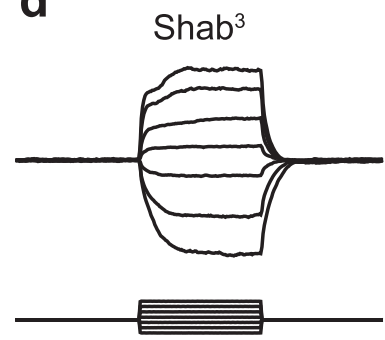

e

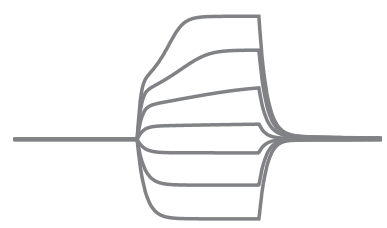

g

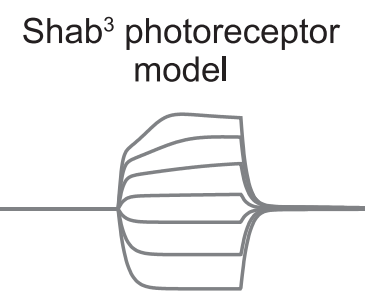

WT photoreceptor
model

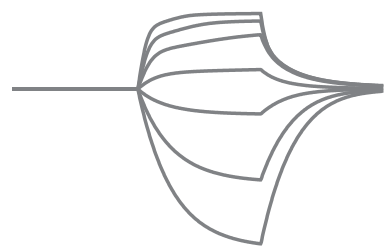

Figure 2. Photo-insensitive membrane properties of WT and Shab ${ }^{3}$ photoreceptors. Experimental data are shown in black and the model simulations are shown in gray throughout the figure. $\boldsymbol{a}$, Intracellular voltage responses to hyperpolarizing and depolarizing current pulses recorded in vivo from a WT photoreceptor. $\boldsymbol{b}$, Simulated voltage responses to current pulses in $\boldsymbol{a}$ after fitting the model to the WT photoreceptor. $c$, Model simulation of voltage responses after removal of the slow delayed rectifier $\mathrm{K}^{+}$conductance from the WT photoreceptor. $\boldsymbol{d}$, IntracelIular voltage responses to hyperpolarizing and depolarizing current pulses recorded in vivo from a Shab ${ }^{3}$ photoreceptor. $\boldsymbol{e}$, Model simulations of the voltage responses to current pulses (same as in $\boldsymbol{c}, \boldsymbol{d})$ after fitting the passive membrane properties of the model in c to the Shab ${ }^{3}$ photoreceptor in $\boldsymbol{d}$. Simulated voltage responses to corresponding current pulses of $S h a b^{3}(\boldsymbol{f})$ and WT photoreceptors $(\boldsymbol{g})$ after incorporating the novel voltage-gated $\mathrm{K}^{+}$conductance and the fractional failure of Shaker $\mathrm{K}^{+}$channel inactivation.

those photoreceptors lacking the fast delayed rectifier, appeared to be the only remaining voltage-gated $\mathrm{K}^{+}$current (Fig. $3 b$ ). This novel conductance generated currents of $\sim 100-200 \mathrm{pA}$ at $0 \mathrm{mV}$ with conspicuously slow activation kinetics (voltage-dependent time constants in the range of $\sim 50-200 \mathrm{~ms}$ ). It was first activated at voltages above approximately $-40 \mathrm{mV}$, with a $V_{50}$ of -11.3 $\mathrm{mV}$ (Boltzmann slope, 12.8) (Fig. 3c), and showed no sign of 
a

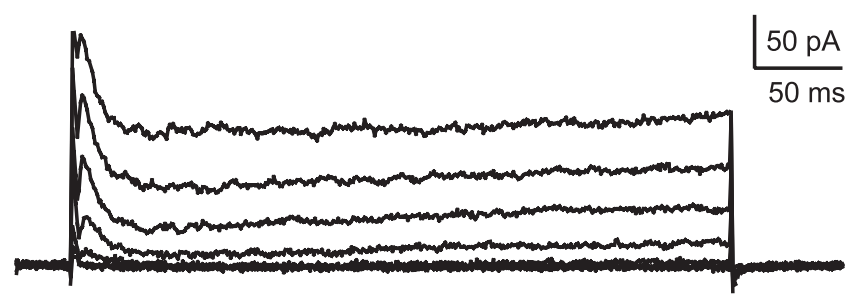

b
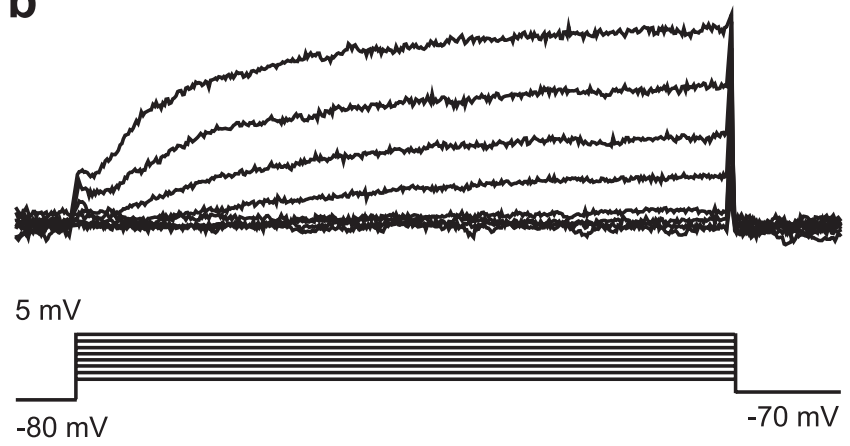

C
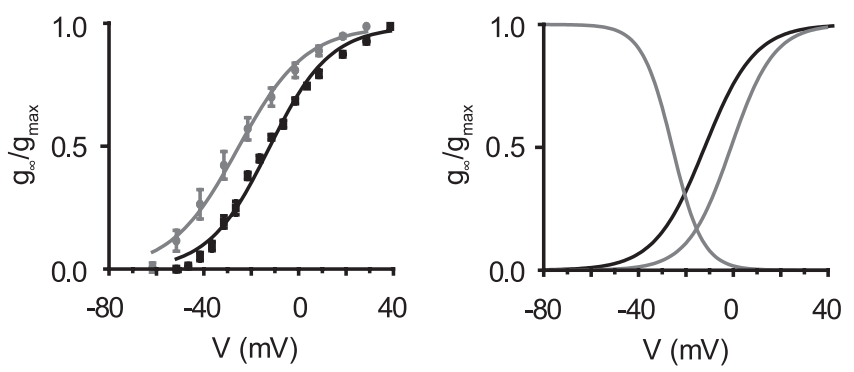

e

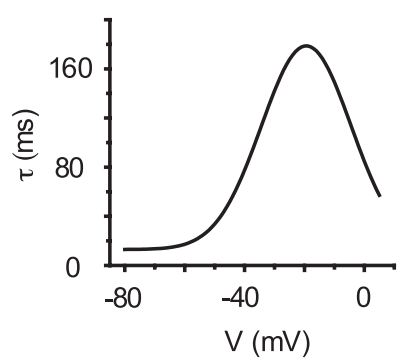

f

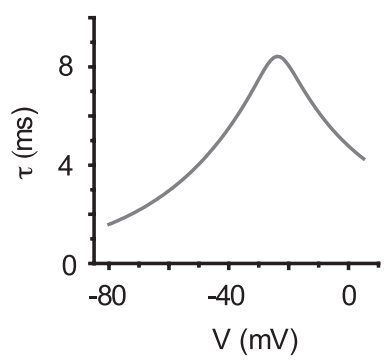

Figure 3. Characterization of a novel slowly activating voltage-gated $\mathrm{K}^{+}$conductance. $\boldsymbol{a}$, Currents recorded in vitro from an isolated Shaker ${ }^{14}$; Shab ${ }^{3}$ double-mutant photoreceptor in which the fast delayed rectifier potassium channel is present. $\boldsymbol{b}$, Currents recorded in vitro from an isolated Shaker ${ }^{14}$; Shab ${ }^{3}$ double-mutant photoreceptor in which the fast delayed rectifier potassium channel is absent. $c$, Voltage dependence of the slowly activating novel conductance in Shaker ${ }^{14}$; Shab ${ }^{3}$ double mutants (black; mean \pm SEM; $n=3-7$ ) and of the non-inactivating component of the current in Shab ${ }^{3}$ mutants (gray; $n=6$ ). Data are fitted with the Boltzmann distribution $\left(V_{50}\right.$ of -11.3 and slope of 12.8 for the Shaker ${ }^{14} ; S$ Shb $^{3} ; V_{50}$ of -24.1 and slope of 14.1 for the Shab ${ }^{3}$ ). $\boldsymbol{d}$, Steady-state activation curve for the novel voltage-gated $\mathrm{K}^{+}$conductance (black) and activation/inactivation curves for the delayed rectifier conductance encoded by Shab (gray).e, Experimentally fitted time constant of activation for the novel voltage-gated $\mathrm{K}^{+}$conductance (for details of fitting the time constants, see Niven et al., 2003a). $\boldsymbol{f}$, Time constant of activation for the delayed rectifier conductance encoded by Shab (gray).

inactivation over at least $5 \mathrm{~s}$. We characterized the voltagedependent properties of this conductance (Fig. 3c,e) and incorporated it into our model.

Although introduction of the novel voltage-gated $\mathrm{K}^{+}$conductance improved the fits between simulated and experimental voltage responses, some discrepancy still remained (data not shown). Examination of $\mathrm{Shab}^{3}$ photoreceptor whole-cell currents (Fig. 1c) revealed that the steady-state current $(>500 \mathrm{~ms}$ after voltage steps to $0 \mathrm{mV}$ ) was at least two times greater than in Shaker ${ }^{14}$;Shab ${ }^{3}$ double mutants $\left(480 \pm 70 \mathrm{pA}, n=5\right.$ in Shab ${ }^{3}$ compared with $180 \pm 78 \mathrm{pA}, n=7$ in Shaker ${ }^{14}$;Shab ${ }^{3}$ ). Furthermore, the voltage dependency of this component in Shab (activated with a $V_{50}$ of $-24 \mathrm{mV}$; slope, 14) (Fig. $3 c$ ) was more negative than that of the novel voltage-gated $\mathrm{K}^{+}$conductance but essentially identical to that of the photoreceptor Shaker channels (Hevers and Hardie, 1995), suggesting that it was dominated by a fraction $(\sim 18 \%)$ of the Shaker channels failing to inactivate. After also incorporating this feature into the model, correspondingly named "Shab $b^{3}$ photoreceptor model," we were able to reproduce the $S h a b^{3}$ voltage responses over the entire voltage range (Fig. 2d,f). WT photoreceptor voltage responses to current steps resimulated with our refined model including the novel conductance and the partial inactivation of the Shaker channels, correspondingly named "WT photoreceptor model," were not significantly altered (Fig. $2 b, g$ ). The relatively small effect of these new features on the WT responses are attributable to their relatively small macroscopic conductance compared with that of the slow delayed rectifier (Figs. 1b,c, 3b). Additionally, the voltage range of activation for the novel conductance overlaps with that of the Shab channels (Fig. $3 d$ ) and has slower activation kinetics (Fig. $3 e, f)$. Therefore, in WT photoreceptor voltage responses, the effect of the novel conductance and the partial inactivation of the Shaker channels are masked by the dominant Shab conductance.

The electrical properties of Shab mutant photoreceptors are similar to WT at physiological voltages, maintaining the light-voltage relationship

Comparison of the voltage responses of WT and Shab ${ }^{3}$ mutant photoreceptors to hyperpolarizing current steps showed that, in addition to the loss of the Shab conductance, Shab ${ }^{3}$ photoreceptors had reduced steady-state input resistances (Fig. 2a,d). How does the decrease in the input resistance affect the electrical properties of Shab ${ }^{3}$ photoreceptors? We used the WT photoreceptor model to predict the $I-V$ relationship of WT photoreceptors for 100 ms current pulses (Fig. 4a) (see Materials and Methods). The duration of these pulses was long enough for Shaker channels to reach steady state, enabling us to separate their effects on the photoreceptor voltage responses from those of the Shab channels. Below the resting potential, the WT $I-V$ relationship was linear. At more depolarized voltages (approximately $-40 \mathrm{mV}$ ), there was a strong rectification attributable to the Shab conductance.

We removed the Shab conductance from the WT photoreceptor model, correspondingly named WT without Shab model, and determined the $I-V$ relationship for this hypothetical case (i.e., no accompanying change in the input resistance) (Fig. $4 a$, light gray dashed line). This $I-V$ relationship deviated strongly from the $I-V$ relationship of the WT photoreceptor model at voltages above $-40 \mathrm{mV}$ (Fig. 4a). The remaining rectification in the WT without Shab model was attributable to the activation of the novel voltage-gated $\mathrm{K}^{+}$conductance and the partial failure of inactivation of the Shaker $\mathrm{K}^{+}$channels. In contrast, the experimentally fitted $S h a b^{3}$ photoreceptor model was close to linear over its entire range (Fig. $4 b$, experimental data points for the WT 
a

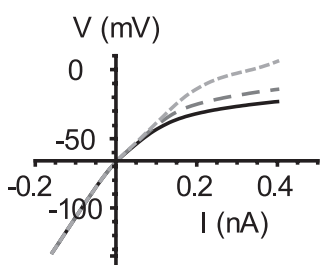

b

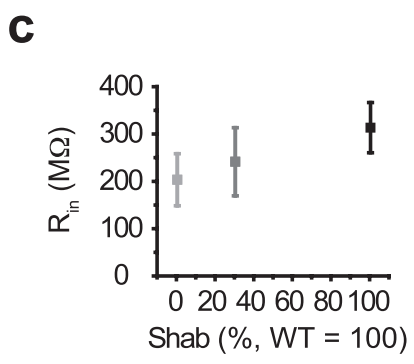

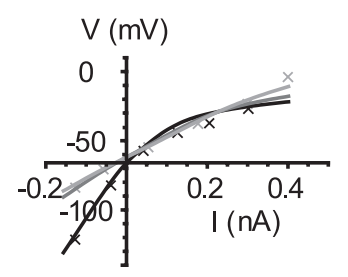

d

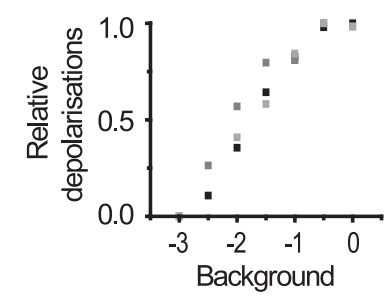

Figure 4. The light-voltage relationship is maintained by regulating the electrical properties of Shab mutant photoreceptors. $a, I-V$ relationships for the WT (black), the WT with $30 \%$ Shab (dark gray, dashed), and the WT without Shab (light gray, dashed) models. b, I-V relationships for the WT (black), Shab ${ }^{1}$ (dark gray), and Shab ${ }^{3}$ (light gray) photoreceptor models. Experimental data points for the WT (black) and $\mathrm{Shab}^{3}$ (light gray) photoreceptors from Figure 2 are shown as crosses. c, Experimentally determined input resistance (mean \pm SD; square) of Shab ${ }^{3}$ (light gray), Shab ${ }^{1}$ (dark gray), and WT (black) photoreceptors under dark-adapted conditions ( $n=15-24)$. $\boldsymbol{d}$, Mean steady-state potentials at different adapting light backgrounds for WT (black), Shab ${ }^{1}$ (dark gray), and Shab ${ }^{3}$ (light gray) photoreceptors normalized to background $-1 . n=2-11$.

and $S h a b^{3}$ are shown as crosses). Despite being linear, the $S h a b^{3}$ photoreceptor model $I-V$ relationship closely matched that of the WT photoreceptor model over most of the physiological voltage range of the photoreceptor $(-70$ to $-15 \mathrm{mV})$. These results suggest that the decreased steady-state input resistance of the Shab ${ }^{3}$ photoreceptors ensures that their $I-V$ relationship remains close to that of the WT photoreceptors (Fig. $4 a, b$ ).

To investigate the relationship between steady-state input resistance and the Shab conductance, we performed additional intracellular experiments from photoreceptors in $S h a b^{1}$ mutants, which have an $\sim 70 \%$ reduction in Shab conductance (Hegde et al., 1999) (experiments were also performed on Shab ${ }^{2}$ mutants but because there were no significant differences between the responses of these two photoreceptor types only Shab $b^{1}$ photoreceptors are discussed further). Following similar procedures as with the WT and $S h a b^{3}$ photoreceptors, we also developed models for $S h a b^{1}$ mutant photoreceptors: a WT with 30\% Shab model generated by removing $70 \%$ of the Shab conductance from the WT photoreceptor model and an experimentally fitted Shab ${ }^{1}$ photoreceptor model. In contrast to the WT with $30 \%$ Shab model (Fig. $4 a$, gray dashed line), the $I-V$ relationship of the $S h a b^{1}$ photoreceptor model closely matched those of simulated WT and $S h a b^{3}$ photoreceptors over the physiological voltage range ( -70 to $-15 \mathrm{mV}$ ) (Fig. $4 b$ ). These findings suggest that the steady-state input resistance decreases in proportion to the reduction of the Shab conductance and that these changes help to maintain the electrical properties of the photo-insensitive membrane of the Shab mutants.

To quantify the observed decrease in the steady-state input resistance, we calculated its mean values in dark for the WT, $S h a b^{1}$, and $S h a b^{3}$ photoreceptors (Fig. $4 c$ ). Shab ${ }^{1}$ and $S h a b^{3}$ photoreceptors had input resistances that were significantly reduced from that of the WT (mean $\pm \mathrm{SD}$ ): WT, $312 \pm 53.4 \mathrm{M} \Omega$; Shab ${ }^{1}$,

$240.0 \pm 72.4 \mathrm{M} \Omega ; \mathrm{Shab}^{3}, 202.2 \pm 54.8 \mathrm{M} \Omega$ (ANOVA, $p<0.05$; $n=15-24$; equal variances tested with Levene's test). Although $S h a b^{3}$ photoreceptors showed additional decrease in their mean input resistance from that of the Shab ${ }^{1}$ photoreceptors, this difference was not significant in our dataset (Bonferroni's test, $p<$ $0.05)$. To determine whether these changes in the input resistance contribute to maintaining the light-voltage relationship as was suggested by the simulated $I-V$ relationships (Fig. $4 a, b$ ), we compared WT, $S h a b^{1}$, and $S h a b^{3}$ photoreceptors voltage responses over a range of adapting light backgrounds (see Materials and Methods). Over all adapting light backgrounds, the mean steadystate membrane potentials of WT, Shab ${ }^{1}$, and $S h a b^{3}$ photoreceptors showed a similar light dependency (Fig. $4 d$ ). This indicates that at least the steady-state properties of the mutant photoreceptors were maintained through the regulation of the electrical properties.

\section{Robustness of dynamic coding of light intensities in Shab mutant photoreceptors}

How do changes in Shab mutant photoreceptors affect their processing of dynamically fluctuating light stimuli? We recorded the voltage responses of WT, $S h a b^{1}$, and $S h a b^{3}$ photoreceptors while presenting repetitions of a white-noise-modulated light contrast stimulus (Fig. $5 a$ ). We also wanted to simulate these experiments to be able to separate the effect of reduced Shab conductance from that of the reduced input resistance in our analysis. For these simulations, we needed a realistic approximation of the phototransduced currents, which could be used as an input to the models. Instead of developing a detailed description of the phototransduction, we reconstructed the LIC from the experimental voltage response of WT photoreceptor (Fig. $5 a$ ) using our photoreceptor models (Fig. 5b) (see Materials and Methods). This reconstructed LIC enabled us to perform realistic simulations of the white-noise experiments.

A linear estimate of the dynamic performance of the photoreceptors can be determined by calculating their frequency response function to light, a measure of light contrast to voltage gain at each frequency. We simulated the dynamic light contrast experiments for our two models of hypothetical photoreceptors (WT with 30\% Shab and WT without Shab models) and calculated their frequency response functions (Fig. 5c). The WT with $30 \%$ Shab and WT without Shab models showed clear increase in their gain at frequencies below $30 \mathrm{~Hz}$ (Fig. $5 c$, highlighted region). This increase was restricted to the frequencies defined by the activation kinetics of the Shab channels. In contrast, the gain functions determined from experimental recordings of the Shab and $S h a b^{3}$ photoreceptors showed no increase (Fig. $5 d$ ). We determined the contrast gains for the experimentally fitted WT, $S h a b^{1}$, and $S h a b^{3}$ photoreceptor models to test whether the decreased steady-state input resistance could explain the difference. The resulting gain functions closely matched the experimental ones (Fig. $5 d, e$ ), indicating that the sensitivity of the photoreceptors to dynamic light intensity fluctuations were maintained in $S h a b^{1}$ and $S h a b^{3}$ photoreceptors by the decreased input resistance.

Photoreceptor performance is constrained not only by the signal gain but also by the noise. We approximated the photoreceptor noise (Fig. $6 a$ ) by removing the mean voltage signal (Fig. $5 a$ ) from individual voltage responses at each light intensity background (see Materials and Methods), allowing comparison of the signal and noise in WT, $S h a b^{1}$, and $S h a b^{3}$ photoreceptors (Figs. $5 a, 6 a, b)$. To quantify the reliability of contrast coding, we decided to use the mean information capacity as a measure of the 
signal-to-noise ratio. Visual inspection of the noise waveforms (Fig. 6a,b) gives an impression of higher noise content in Shab mutant photoreceptors, which would be expected to reduce their information capacities. However, examination of the frequency dependence of information in WT, $S h a b^{1}$, and $S h a b^{3}$ photoreceptors revealed that $S h a b^{3}$ photoreceptors had less information at low frequencies, at which Shab channels contribute to the information processing (Fig. $5 c$ ), but more information at high frequencies compared with WT photoreceptors (Fig. $6 c$ ). Therefore, the mean information capacities of WT, $S h a b^{1}$, and $S h a b^{3}$ mutant photoreceptors were similar over different light intensity backgrounds (Fig. 6d). The increased information content at the high frequencies can be explained by the average signal content, which extends to higher frequencies in Shab mutant photoreceptors (Fig. $5 d$ ). These results suggest that robustness of the function against imposed changes in specific ion channel composition extends from the static light-voltage relationship to the dynamic coding of light intensities.

\section{Discussion}

We have shown that the Shab gene encodes the slow delayed rectifier voltage-gated $\mathrm{K}^{+}$ channels in Drosophila photoreceptors. We investigated the functional role of this channel by using a combination of in vivo and in vitro recordings and model simulations. Three alleles of the Shab gene (Hegde et al., 1999) (Shab ${ }^{1}$, Shab ${ }^{2}$, and $S h a b^{3}$ ) were used to eliminate $\sim 75,71$, and $100 \%$ of the Shab conductance, respectively. Using HodgkinHuxley-type modeling, we predicted the effect of these alleles on the photo-insensitive membrane of Drosophila photoreceptors: a dramatic increase in steady-state depolarization and gain at a particular light background but no change in input resistance in the dark. However, all of these alleles produced an unexpected result: they showed little or no change in steady-state depolarization or gain in the light. This appears to be attributable to decreased input resistance, coupled to the decrease in Shab conductance, which restores their light-voltage relationships and sensitivity to resemble those of the WT photoreceptors over the physiological voltage range.

The decreased input resistance associated with deletion of the Shab channels is similar to that found in Shaker mutant photoreceptors (Niven et al., 2003a), although it is unclear whether the underlying mechanisms are the same. Despite similarities, however, these two types of photoreceptor show robustness in different aspects of their function. In Shaker mutant photoreceptors, the decrease in input resistance partially restores the efficient use of the operating voltage range (Niven et al., 2003a). Shab mutant photoreceptors, conversely, show remarkable robustness in their light-voltage relationships, sensitivities (light contrast gains), and reliability of dynamic coding (information capacities). The decreased input resistance in the Shab mutant photoreceptors significantly improved these aspects of their performance, but b

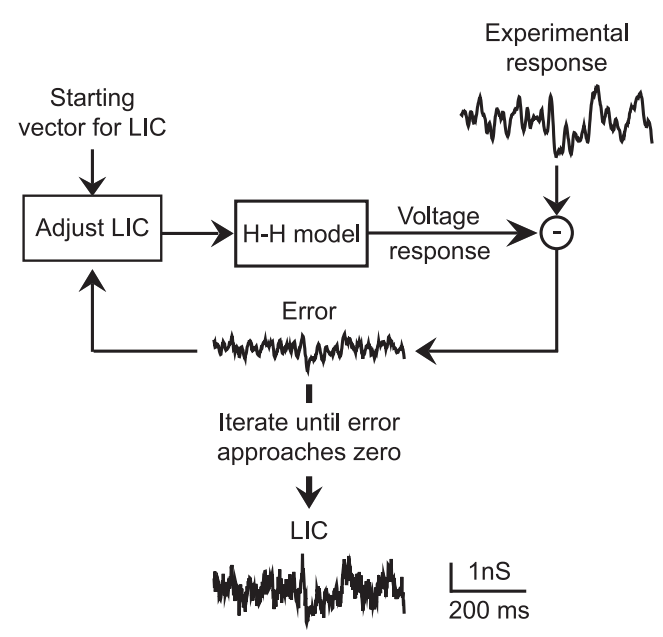

d

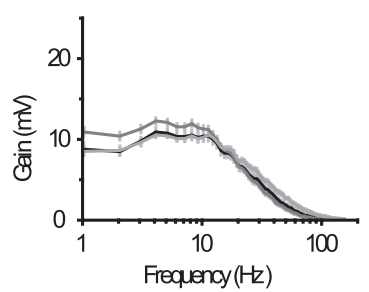

e

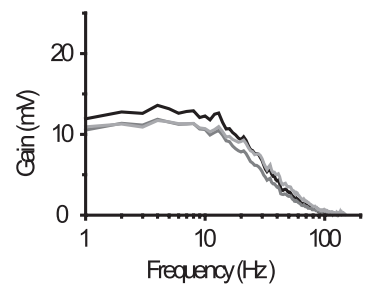

Figure 5. Reduced input resistance maintains the contrast gain of Shab mutant photoreceptor. $\boldsymbol{a}$, Waveforms of the average voltage signal of WT, Shab ${ }^{1}$, and $S h a b^{3}$ photoreceptors to a white-noise-modulated light contrast stimulus at the background -1 $S h a b^{1}$ (dark gray), and Shab ${ }^{3}$ (light gray) photoreceptors at background -1. $n=6$. e, Simulated frequency response functions, $G(f)$, for the WT (black), Shab ${ }^{7}$ (dark gray), and Shab ${ }^{3}$ (light gray) photoreceptor models. The same LIC was used as in $\mathbf{c}$.

clear disadvantages may also follow from the deletion of the voltage-gated channels.

Drosophila photoreceptors, like those of other insects, generate a maintained depolarization in response to increasing light intensity (Weckström and Laughlin, 1995). Because of their voltage dependency (Hardie, 1991a; Hevers and Hardie, 1995; Niven et al., 2003a), Shab channels open at high light intensities, thereby attenuating the light-induced depolarization and preventing response saturation. In addition, the activation of Shab channels at high light intensities decreases the membrane time constant, allowing photoreceptors to encode faster events (Weckström and Laughlin, 1995). Although these general effects of the $\mathrm{K}^{+}$channels were reproduced by the increased nonspecific background conductance, a significant decrease in the coding efficiency of the photoreceptors is to be expected. Modeling has provided evidence that the maintenance of the resting potential when photoreceptors are in the dark is energetically expensive (Laughlin et al., 1998; Laughlin, 2001; Niven et al., 2003b). Few Shab channels will be active in the dark, contributing relatively little to the input resistance (Hardie, 1991a; Hevers and Hardie, 1995; Niven et al., 2003a). In contrast to the Shab channels, which are activated only in response to relatively large depolarizing events, the decreased input resistance in Shab mutants imposes a constant, high-energy cost to the photoreceptors. Therefore, Shab mutant photoreceptors are likely to consume significantly more energy in the dark or at low light levels than the WT photoreceptors. 
a
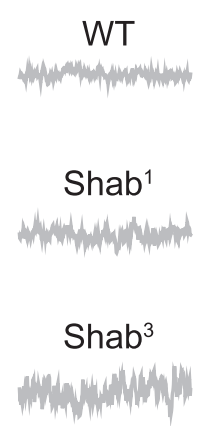

C

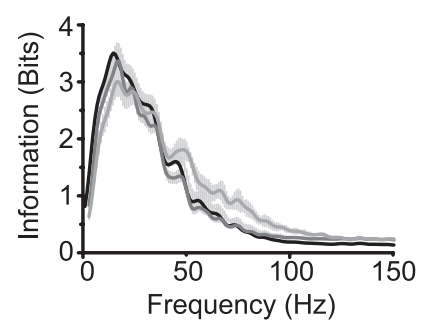

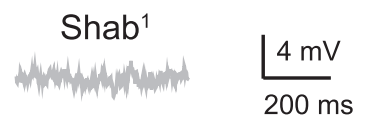

b
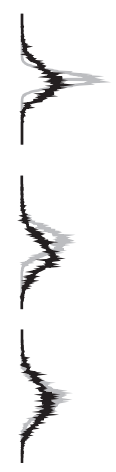

d

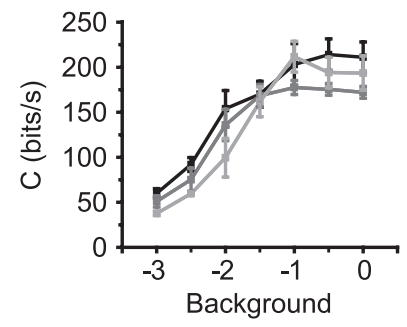

Figure 6. Robustness of contrast coding in Shab mutant photoreceptors. $\boldsymbol{a}$, The corresponding voltage noise for the average signals (see Fig. 5a) in WT, Shab ${ }^{1}$, and Shab ${ }^{3}$ photoreceptors at background $-1 . \boldsymbol{b}$, Distributions of the signal (black) and noise (gray). $\boldsymbol{c}$, The mean \pm SE frequency dependence of information extracted from the light contrast stimulus in WT (black), Shab ${ }^{1}$ (dark gray), and Shab ${ }^{3}$ (light gray) photoreceptors at background - 0.5. d, Information capacity, C (the integral of the information rate over frequencies up to $200 \mathrm{~Hz}$ ) for the WT (black), Shab ${ }^{1}$ (dark gray), and $S h a b^{3}$ (light gray) photoreceptors over a range of light intensities up to $3 \times 10^{6}$ photons/s. Mean \pm SD is shown $(n=3-15)$.

In addition to reducing energetic expenditure, the kinetics of Shab channels also contribute to the nonlinear processing of natural stimuli, which contain strong temporal fluctuations, large changes in intensity, and a higher proportion of low-frequency signals (Field, 1987; Tolhurst et al., 1992; Ruderman and Bialek, 1994; van Hateren, 1997). We have shown previously that the effects of dynamic nonlinearities generated by voltage-gated Shaker channels are important for the performance of Drosophila photoreceptors in coding naturalistic stimuli (Niven et al., 2004). Such nonlinear effects of voltage-gated $\mathrm{K}^{+}$channels cannot be reproduced by changes in input resistance, which is close to a linear effect, or by an increase in the magnitude of other voltagegated conductances, whose kinetic properties are different. Although the nonlinear characterization of the Shab channels during natural stimuli is beyond the scope of the present work, we suggest that they are essential for tuning the membrane properties of the photoreceptors to their input statistics and dynamics. Despite a reduction in the amount of information at low frequencies, the information capacities of $S h a b^{3}$ photoreceptors exposed to white-noise stimuli were close to those of WT Drosophila photoreceptors because of an increase in the information at high frequencies. However, when processing natural images with a high proportion of low-frequency signal content, Shab mutant photoreceptors may have significantly lower information capacities than their WT counterparts.

Additional insights into the robustness of neural processing in Drosophila photoreceptors may come from the identification of the genes encoding other voltage-gated $\mathrm{K}^{+}$channels. Our results suggest that at least two more voltage-gated $\mathrm{K}^{+}$channels remain to be identified genetically: the novel non-inactivating channel we have characterized and the fast delayed rectifier channel, which had been characterized previously (Hardie, 1991a). These conductances are not encoded by either the Shab or Shaker genes and are likely to be formed from separate gene products, not heteromultimers (Covarrubias et al., 1991). The Shal gene would seem the most likely candidate for the fast-inactivating voltagegated $\mathrm{K}^{+}$channels because its properties are similar to those reported for Shal channels identified in other tissues and when heterogously expressed (Wei et al., 1990; Pak et al., 1991). The identity of the novel non-inactivating channel is less clear. In oocyte expression studies, both Shaw, the remaining member of the Shaker-like gene family in Drosophila (Butler et al., 1989; Wei et al., 1990), and eag (ether-a-go-go) are reported to encode noninactivating $\mathrm{K}^{+}$conductances (Wei et al., 1990; Bruggemann et al., 1993; Robertson et al., 1996; Martinez-Padron and Ferrus, 1997), but both have rapid activation kinetics inconsistent with the slow activation observed for the residual current in Shaker ${ }^{14}$; $S h a b^{3}$ double mutants. However, mutant and/or overexpression studies of these candidates in the photoreceptors are needed before ruling them out with certainty.

What could be the possible neuronal mechanism behind the decrease in the input resistance? This decrease was reproduced in the models by increasing the leak conductances. In HodgkinHuxley-type models, the leak conductances are routinely used to cover all of the undefined processes required to fit the models to the experimental data. Thus, leak conductances can be considered as a descriptive representation of possibly several neuronal mechanisms. A recent study has demonstrated that electrical properties may be restored by the compensatory increase in $\mathrm{K}^{+}$ leak conductance in cerebellar granule cells in which a tonic synaptic conductance was eliminated (Brickley et al., 2001). Whether the leak conductances in our models have cellular counterparts or whether they describe overall effect of more complicated processes remains to be determined in future work.

Our results show that regulation of the electrical properties maintains the light-voltage relationship in Drosophila photoreceptors after imposed changes in their ion channel composition. This relates closely to recent studies in which robustness of electrical properties is shown to optimize information processing by maintaining the dynamic range of signaling within useful limits (Stemmler and Koch, 1999; Turrigiano and Nelson, 2004). However, it is also clear that certain features of voltage-gated ion channels cannot be fully restored by concurrent changes in the passive membrane properties or by upregulation or downregulation of other ion channels (Niven et al., 2003a,b; Niven, 2004). Understanding the mechanisms and limitations of robustness of neural coding could have important implications for the evolution of nervous systems, especially when addressing why specific ion channels are expressed in specific neurons.

\section{References}

Akopian AN, Souslova V, England S, Okuse K, Ogata N, Ure J, Smith A, Kerr BJ, Mc Mahon SB, Boyce S, Hill R, Stanfa LC, Dickenson AH, Wood JN (1999) The tetrodotoxin-resistant sodium channel SNS has a specialized function in pain pathways. Nat Neurosci 2:541-548.

Bernard C, Anderson A, Becker A, Poolos NP, Beck H, Johnston S (2004) Acquired dendritic channelopathy in temporal lobe epilepsy. Science 305:532-535.

Brickley SG, Revilla V, Cull-Candy SG, Wisden W, Farrant M (2001) Adaptive regulation of neuronal excitability by a voltage-independent potassium conductance. Nature 409:88-92.

Bruggemann A, Pardo LA, Stuhmer W, Pongs O (1993) Ether-a-go-go en- 
codes a voltage-gated channel permeable to $\mathrm{K}^{+}$and $\mathrm{Ca}^{2+}$ and modulated by cAMP. Nature 365:445-448.

Butler A, Wei AG, Baker K, Salkoff L (1989) A family of putative potassium channel genes in Drosophila. Science 243:943-947.

Coetzee WA, Amarillo Y, Chiu J, Chow A, Lau D, McCormack T, Moreno H, Nadal MS, Ozaita A, Pountney D, Saganich M, Vega-Saenz de Miera E, Rudy B (1999) Molecular diversity of $\mathrm{K}^{+}$channels. Ann NY Acad Sci 868:233-285.

Connor JA, Stevens CF (1971) Voltage clamp studies of a transient outward membrane current in gastropod neural somata. J Physiol (Lond) 213:21-30.

Covarrubias M, Wei AA, Salkoff L (1991) Shaker, Shal, Shab, and Shaw express independent $\mathrm{K}^{+}$current systems. Neuron 7:763-773.

Debanne D, Guerineau NC, Gahwiler BH, Thompson SM (1997) Actionpotential propagation gated by an axonal $\mathrm{I}_{\mathrm{A}}$-like $\mathrm{K}^{+}$conductance in hippocampus. Nature 389:286-289.

Field DJ (1987) Relations between the statistics of natural images and the response properties of cortical cells. J Opt Soc Am A 4:2379-2394.

Franceschini N, Kirschfeld K (1971) Etude optique in vivo des éléments photorecepteurs dans l'oeil composé de Drosophila. Kybernetik 9:159-182.

Goldman MS, Golowasch J, Marder E, Abbott LF (2001) Global structure, robustness, and modulation of neuronal models. J Neurosci 21:5229-5238.

Golowasch J, Abbott LF, Marder E (1999) Activity-dependent regulation of potassium currents in an identified neuron of the stomatogastric ganglion of the crab Cancer borealis. J Neurosci 19:RC33(1-5).

Hardie RC (1991a) Voltage-sensitive potassium channels in Drosophila photoreceptors. J Neurosci 11:3079-3095.

Hardie RC (1991b) Whole-cell recordings of the light-induced current in dissociated Drosophila photoreceptors: evidence for feedback by calcium permeating the light-sensitive channels. Proc R Soc Lond B Biol Sci 245:203-210.

Hardie RC, Voss D, Pongs O, Laughlin SB (1991) Novel potassium channels encoded by the Shaker locus in Drosophila photoreceptors. Neuron 6:477-486.

Hegde P, Gu GG, Chen D, Free SJ, Singh S (1999) Mutational analysis of the Shab-encoded delayed rectifier $\mathrm{K}^{+}$channels in Drosophila. J Biol Chem 274:22109-22113.

Henderson SR, Reuss H, Hardie RC (2000) Single photon responses in Drosophila photoreceptors and their regulation by $\mathrm{Ca}^{2+}$. J Physiol (Lond) 524:179-194.

Hevers W, Hardie RC (1995) Serotonin modulates the voltage dependence of delayed rectifier and Shaker potassium channels in Drosophila photoreceptors. Neuron 14:845-856.

Hille B (2001) Ion channels of excitable membranes. Sunderland, MA: Sinauer.

Hoffman DA, Magee JC, Colbert CM, Johnston D (1997) $\mathrm{K}^{+}$channel regulation of signal propagation in dendrites of hippocampal pyramidal neurons. Nature 387:869-875.

Howard J, Blakeslee B, Laughlin SB (1987) The intracellular pupil mechanism and photoreceptor signal: noise ratios in the fly Lucilia cuprina. Proc R Soc Lond B Biol Sci 231:415-435.

Juusola M, de Polavieja GG (2003) The rate of information transfer of naturalistic stimulation by graded potentials. J Gen Physiol 122:191-206.

Juusola M, Hardie RC (2001) Light adaptation in Drosophila photoreceptors. I. Response dynamics and signaling efficiency at 25 degrees C. J Gen Physiol 117:3-25.

Laughlin SB (2001) Energy as a constraint on the coding and processing of sensory information. Curr Opin Neurobiol 11:475-480.

Laughlin SB, de Ruyter van Steveninck RR, Anderson JC (1998) The metabolic cost of neural information. Nat Neurosci 1:36-41.

Laurent G (1990) Voltage-dependent nonlinearities in the membrane of lo- cust nonspiking local interneurons, and their significance for synaptic integration. J Neurosci 10:2268-2280.

Liu Z, Golowasch J, Marder E, Abbott LF (1998) A model neuron with activity-dependent conductances regulated by multiple calcium sensors. J Neurosci 18:2309-2320.

MacLean JN, Zhang Y, Johnson BR, Harris-Warrick RM (2003) Activityindependent homeostasis in rhythmically active neurons. Neuron 37:109-120.

Marder E, Prinz AA (2002) Modeling stability in neuron and network function: the role of activity in homeostasis. BioEssays 24:1145-1154.

Martinez-Padron M, Ferrus A (1997) Presynaptic recordings from Drosophila: correlation of macroscopic and single-channel $\mathrm{K}^{+}$currents. J Neurosci 17:3412-3424.

Namkung Y, Smith SM, Lee SB, Skrypnyk NV, Kim HL, Chin H, Scheller RH, Tsien RW, Shin HS (1998) Targeted disruption of the $\mathrm{Ca}^{2+}$ channel beta3 subunit reduces $\mathrm{N}$ - and L-type $\mathrm{Ca}^{2+}$ channel activity and alters the voltage-dependent activation of $\mathrm{P} / \mathrm{Q}-$ type $\mathrm{Ca}^{2+}$ channels in neurons. Proc Natl Acad Sci USA 95:12010-12015.

Niven JE (2004) Channelling evolution: canalization and the nervous system. PLoS Biol 2:E19.

Niven JE, Vähäsöyrinki M, Kauranen M, Hardie RC, Juusola M, Weckström M (2003a) The contribution of Shaker $\mathrm{K}^{+}$channels to the information capacity of Drosophila photoreceptors. Nature 421:630-634.

Niven JE, Vähäsöyrinki M, Juusola M (2003b) Shaker $\mathrm{K}^{+}$-channels are predicted to reduce the metabolic cost of neural information in Drosophila photoreceptors. Proc R Soc Lond B Biol Sci 270:S58-S61.

Niven JE, Vähäsöyrinki M, Juusola M, French AS (2004) Interactions between light-induced currents, voltage-gated currents, and input signal properties in Drosophila photoreceptors. J Neurophysiol 91:2696-2706.

Pak MD, Baker K, Covarrubias M, Butler A, Ratcliffe A, Salkoff L (1991) mShal, a subfamily of A-type $\mathrm{K}^{+}$channel cloned from mammalian brain. Proc Natl Acad Sci USA 88:4386-4390.

Prinz AA, Billimoria CP, Marder E (2003) Alternative to hand-tuning conductance-based models: construction and analysis of databases of model neurons. J Neurophysiol 90:3998-4015.

Reuss H, Mojet MH, Chyb S, Hardie RC (1997) In vivo analysis of the drosophila light-sensitive channels, TRP and TRPL. Neuron 19:1249-1259.

Robertson GA, Warmke JM, Ganetsky B (1996) Potassium currents expressed from Drosophila and mouse eag cDNAs in Xenopus oocytes. Neuropharmacology 35:841-850.

Ruderman DL, Bialek W (1994) Statistics of natural images: scaling in the woods. Phys Rev Lett 73:814-817.

Rudy B (1988) Diversity and ubiquity of K channels. Neuroscience 25:729-749.

Stemmler M, Koch C (1999) How voltage-dependent conductances can adapt to maximize the information encoded by neuronal firing rate. Nat Neurosci 2:521-527.

Tolhurst DJ, Tadmor Y, Chao T (1992) Amplitude spectra of natural images. Ophthalmic Physiol Opt 12:229-232.

Turrigiano GG, Nelson SB (2004) Homeostatic plasticity in the developing nervous system. Nat Rev Neurosci 5:97-107.

van Hateren JH (1997) Processing of natural time series of intensities by the visual system of the blowfly. Vision Res 37:3407-3416.

Weckström M, Laughlin SB (1995) Visual ecology and voltage-gated ion channels in insect photoreceptors. Trends Neurosci 18:17-21.

Weckström M, Hardie RC, Laughlin SB (1991) Voltage-activated potassium channels in blowfly photoreceptors and their role in light adaptation. J Physiol (Lond) 440:635-657.

Wei A, Covarrubias M, Butler A, Baker K, Pak M, Salkoff L (1990) $\mathrm{K}^{+}$ current diversity is produced by an extended gene family conserved in Drosophila and mouse. Science 248:599-603.

Wickman K, Nemec J, Gendler SJ, Clapham DE (1998) Abnormal heart rate regulation in GIRK4 knockout mice. Neuron 20:103-114. 